\title{
Suppression of Multiple Pulse Formation in External-Cavity Mode-Locked Semiconductor Lasers Using Intrawaveguide Saturable Absorbers
}

\author{
D. J. Derickson, R. J. Helkey, A. Mar, J. R. Karin, J. E. Bowers, and R. L. Thornton
}

\begin{abstract}
Imperfect antireflection coatings in external-cavity mode-locked semiconductor lasers can cause multiple output pulse generation. The incorporation of an intrawaveguide saturable absorber segment into the laser suppresses this problem. Single pulse outputs of less than $2.8 \mathrm{ps}$ and $0.7 \mathrm{pJ}$ of energy are obtained using such devices with both quantum well and bulk active regions.
\end{abstract}

$\mathbf{M}^{2}$ ODE-LOCKED semiconductor lasers are important sources of short optical pulses for optical communications, optoelectronic measurement systems, and physics experiments. Active and hybrid active/passive mode-locking are the most important modulation techniques for these applications because of their amplitude and temporal stability [1] and the presence of a system timing reference. However, actively mode-locked external cavity semiconductor lasers with a single gain segment are very susceptible to multiple pulse formation [2]. The undesired secondary pulses are initiated by reflections from imperfect antireflection coatings on the laser-diode facet. The reflected pulse is then amplified because the main pulse does not fully deplete the gain and current drive to the segment may still be creating new carriers. Schell et al. [3] have calculated that even a very good antireflection coating, $10^{-5}$, will cause multiple pulse generation at the round-trip time of the diode laser. In this letter we show that incorporation of reverse-biased intrawaveguide saturable absorber segments [4] into the cavity suppresses the multiple pulse generation problem. The technique is applicable to both bulk and quantum-well active region lasers. Single pulse outputs of less than 2.8 ps with $0.7 \mathrm{pJ}$ of energy are obtained using these techniques.

The device and configuration used to suppress the multiple pulse output problem is shown in Fig. 1. The top contacts of

Manuscript received November 5, 1991. This work was supported by the Office of Naval Research. R. Helkey was supported by a Newport Research Fellowship.

D. J. Derickson, R. J. Helkey, A. Mar, and J. E. Bowers are with the Department of Electrical and Computer Engineering, University of California, Santa Barbara, CA 93106.

R. L. Thornton is with Xerox Palo Alto Research Center, Palo Alto, CA 94304.

IEEE Log Number 9106991.

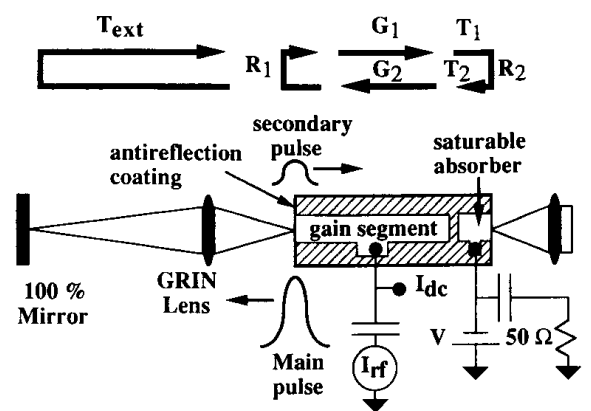

Fig. 1. The external cavity configuration used for testing the two-section mode-locked lasers.

the laser diode are separated to allow sections of the laser to be biased independently. The long segment provides the overall gain for the device. The reverse biased segment acts as an intrawaveguide saturable absorber and $\mathrm{p}-\mathrm{i}-\mathrm{n}$ photodetector. To illustrate how the saturable absorber can suppress multiple pulse formation, the propagation of a pulse around the mode-locked cavity of Fig. 1 is outlined in Fig. 2. The modeled device is a two section laser with a $500 \mu \mathrm{m}$ overall length. The saturation energy, $E_{\text {sat }}$, of a gain or an absorbing segment is defined as [5]

$$
E_{\mathrm{sat}}=\frac{h \nu A}{d g / d n}
$$

where $h \nu$ is the photon energy, $d g / d n$ is the differential gain, and $A$ is the mode cross sectional area. The saturation energy is a measure of the energy required to saturate the gain of a gain section or the absorption of an absorber segment. The $E_{\text {sat }}$ of the gain section is $2.1 \mathrm{pJ}$ and the $E_{\text {sat }}$ of the absorber is $0.7 \mathrm{pJ}$ in this simulation. The single pass unsaturated energy gain of an amplifier segment is 17 and the unsaturated transmission through the absorber is 0.05 . Fig. 2(a) shows rate equation simulations of the pulse energy versus distance for the main pulse as the pulse enters from the external cavity and propagates through the gain and absorber segments on the forward and reverse transits. The energy gain through the first gain segment, $G_{1}$, is only 8.5 


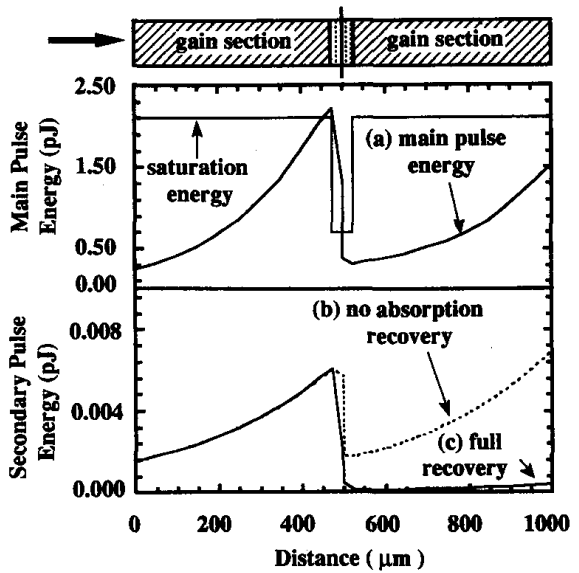

Fig. 2. Simulated pulse energy versus distance along the diode laser for a $500 \mu \mathrm{m}$ long device. (a) The energy versus distance for the main pulse. The energy versus distance for a secondary pulse initiated by an imperfect antireflection coating without absorption recovery (b) and with full absorption recovery (c). The saturation energy versus distance is also shown.

due to large gain saturation. The pulse then encounters the saturable absorber where the leading edge of the pulse is absorbed and the pulse is narrowed. The mirror returns $R_{2}=0.30$ of the energy in the opposite direction. On the return trip through the gain section, the pulse experiences a gain, $G_{2}$, of only 5.0 due to the initial gain depletion from the first gain section transit. The pulse then passes into the external cavity which returns $R_{\text {ext }}=0.15$ of the energy back again to the gain section. The round-trip energy gain, $G_{\pi}$, for the main pulse around the cavity is

$$
G_{\mathrm{rt}}=G_{1} T_{1} R_{2} T_{2} G_{2} R_{\text {ext }}=1
$$

where $T_{1}$ and $T_{2}$ are the forward and reverse transmissions through the saturable absorber. A secondary pulse is initiated by the $R_{1}=10^{-3}$ antireflection coated facet as the main pulse enters the external cavity. The energy versus distance for the secondary pulse is shown in Fig. 2(b) and (c) for the cases of no saturable absorber recovery and complete saturable absorber recovery, respectively. The low energy secondary pulse experiences an unsaturated gain of $G_{1 s}=G_{2 s}$ $=4$. In Fig. 2(b), the absorption does not recover leading to an overall gain of 4.8 through the laser diode. The saturable absorber energy transmission, $T_{\mathrm{ab}}$, decreases as

$$
T_{\mathrm{ab}}(t)=T_{o} e\left[\ln \left(\frac{1}{T_{o}}\right) e\left(\frac{-t}{\tau_{\mathrm{ab}}}\right)\right]
$$

where $T_{o}$ is the unsaturated loss through the absorber, $\tau_{\mathrm{ab}}$ is the carrier density decay constant, and $t$ is the time measured with respect to the passage of the main pulse through the saturable absorber. If the absorber completely recovers, as seen in Fig. 2(c), the secondary pulse is partially absorbed, and the round trip gain for the secondary pulse, $G_{\mathrm{rts}}$, is reduced from 0.72 to 0.036 . If the pulse energies are assumed to add incoherently, the secondary pulse energy, $E_{\mathrm{sec}}$, at the $R_{2}$ facet builds up as

$$
E_{\mathrm{sec}}(I)=E_{\text {main }} R_{1}+G_{\mathrm{rts}} E_{\mathrm{sec}}(I-1)
$$

where $I$ is the round-trip index and $E_{\text {main }}$ is the main pulse energy incident on the $R_{1}$ facet. If $G_{\mathrm{rts}}$ is less than unity, the ratio of the main pulse to secondary pulse energies at the laser output is

$$
\frac{E_{\text {out main }}}{E_{\text {sout sec }}}=\left(\frac{G_{1} T_{1}(0)}{G_{1 s}}\right) \frac{R_{\text {ext }}\left(1-G_{\mathrm{rts}}\right)}{T_{1}\left(t_{\mathrm{rt}}\right) R_{1}} \approx \frac{R_{\mathrm{ext}}\left(1-G_{\mathrm{rts}}\right)}{T_{1}\left(t_{\mathrm{rt}}\right) R_{1}}
$$

where $t_{\mathrm{rt}}$ is the laser diode round trip time. For the examples of Fig. 2(b) and (c), the ratio of main to secondary energies would be 40 for no absorption recovery, and 680 for full absorption recovery. The example of Fig. 2 illustrates that significant secondary pulse build-up can occur if the saturable absorber does not recover in the diode round trip time, $t_{\pi}$. If $G_{\mathrm{rts}}$ is greater than one, the pulse will continually build up until the energy of the secondary pulse saturates the gain enough to reduce the round trip gain back to one. For very short laser diode lengths, two effects increase $G_{\mathrm{rts}}$ : i) the absorption cannot recovery significantly in $t_{\mathrm{rt}}$ and ii) the gain for the secondary pulses increases because the main pulse has not fully depleted the gain before the secondary pulse is initiated. For very long laser-diode lengths, the gain for the secondary pulse recovers significantly from the saturated gain values during the secondary pulse transit leading to a $G_{\text {rs }}$ greater than one.

To test the saturable absorber's ability to suppress secondary pulses, bulk active region devices with gain section lengths of 150,250 , and $500 \mu \mathrm{m}$ and absorber lengths of 8 and $16 \mu \mathrm{m}$ are tested. These lengths correspond to a $t_{\mathrm{rt}}$ of $4.0,6.7$, and $13.3 \mathrm{ps}$ respectively, and estimated $T_{o}$ values of 0.05 and 0.22 , respectively. The bulk active region devices have a $80 \mathrm{~nm}$ GaAs active region with $\mathrm{Al}_{0.4} \mathrm{Ga}_{0.6}$ As cladding layers. Quantum-well devices with four $15 \mathrm{~nm}$ wells and $8.5 \mathrm{~nm}$ thick $\mathrm{Al}_{0.2} \mathrm{Ga}_{0.8} \mathrm{As}$ barriers were tested. The lateral index guide is formed by impurity-induced disordering in both laser types [6]. Isolation between segments is achieved by proton bombardment between segments to provide over 1 $\mathrm{M} \Omega$ of intercontact resistance. To form external cavity lasers, one facet is antireflection coated, and the light is coupled into an external cavity with a graded index lens. The sputtered silicon-nitride antireflection coatings typically have $10^{-3}$ power reflectivities. The devices are passively mode-locked at $5 \mathrm{GHz}$ with $-1.5 \mathrm{~V}$ on the saturable absorber and gain section currents of 48,49 , and $52 \mathrm{~mA}$. The pulses are measured by second harmonic autocorrelation and the resulting traces shown in Fig. 3. This figure shows that for very short device lengths, secondary pulse generation is hard to suppress due to the small absorption recovery and a larger secondary pulse gain. The autocorrelation trace for the $\mathbf{1 5 0}$ $\mu \mathrm{m}$ device showed partially defined secondary pulses, the $250 \mu \mathrm{m}$ device showed highly suppressed secondary pulses and the $500 \mu \mathrm{m}$ device had no detectable secondary pulse. In the frequency domain, multiple pulse generation manifests 


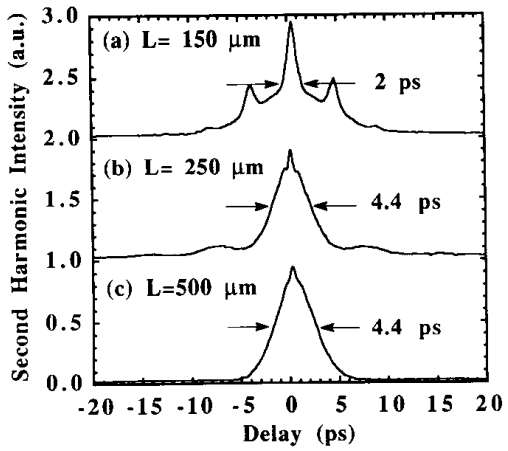

Fig. 3. The second harmonic autocorrelation traces for (a) $150 \mu \mathrm{m}$, (b) 250 $\mu \mathrm{m}$, and (c) $500 \mu \mathrm{m}$ long bulk active region devices with $16 \mu \mathrm{m}$ saturable absorbers.

itself as ripple in the optical spectrum. The ripple frequency spacing is $1 / t_{\mathrm{rt}}$. Fig. 4 shows the progressive reduction in spectral ripple as the secondary pulses are reduced. The 500 $\mu \mathrm{m}$ device produced a $4.4 \mathrm{ps}$ autocorrelation width. If a sech ${ }^{2}$ $(x)$ pulse shape is assumed, the pulses are 2.9 ps wide with a peak power of $0.25 \mathrm{~W}$. A $500 \mu \mathrm{m}$ long quantum well device with an $80 \mu \mathrm{m}$ long saturable absorber gave a single $2.5 \mathrm{ps}$ wide pulse and peak power of $0.25 \mathrm{~W}$. Similar results are obtained when active modulation is added to give better timing stability [1].

The absorption recovery time of the saturable absorber was measured by replacing the $50 \Omega$ saturable absorber termination in Fig. 1 with a high-speed sampling oscilloscope and by pump-probe measurements. The photocurrent waveform from the device of Fig. 3(c) was measured and is shown in Fig. 5(a). Fig. 5(a) shows that absorption can recover very quickly in the saturable absorber which leads to suppression of secondary pulse formation. Pump-probe measurements of the saturable absorber showed that the absorption recovers to a high absorption state in approximately 15 ps. Fig. 5(b) shows the photocurrent for a $1.6 \mathrm{~mm}$ long quantum well device with an $80 \mu \mathrm{m}$ saturable absorber. The photocurrent shows clear absorption peaks spaced at $t_{\mathrm{r}}$. The first pulse is created by the leading edge of the main pulse. The second and third peaks are due to partial absorption of secondary pulses. An external detector measurement of the optical output showed that the energy of the secondary pulse was $15 \%$ of the main pulse. The cause of the secondary pulses in this case is presumably due to significant gain recovery during the secondary pulse transit in this long device.

In summary, it has been shown that multiple pulse behavior can be suppressed with the inclusion of intrawaveguide saturable absorbers into the mode-locked laser. The saturable absorber recovery time constant and the laser-diode round-trip time are important parameters in the effectiveness of the suppression. In addition, the saturable absorption mechanism can produce short pulses with energies approaching the saturation energy of the gain section without the need for fast electrical pumping waveforms. This technique is applicable to lasers with bulk or quantum-well active regions and incorporates easily into many existing lasers processes.

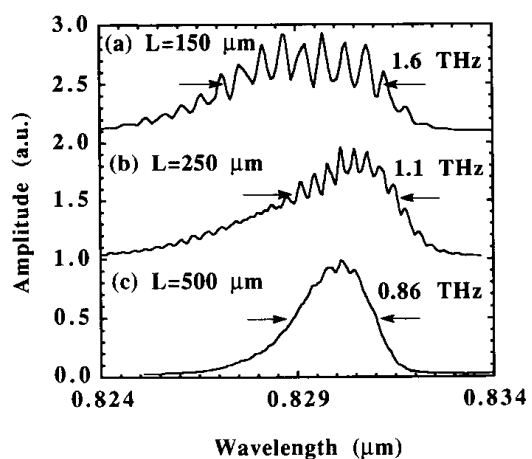

Fig. 4. The optical spectrum for (a) $150 \mu \mathrm{m}$, (b) $250 \mu \mathrm{m}$, and (c) $500 \mu \mathrm{m}$ long bulk active region devices with $16 \mu \mathrm{m}$ saturable absorbers.

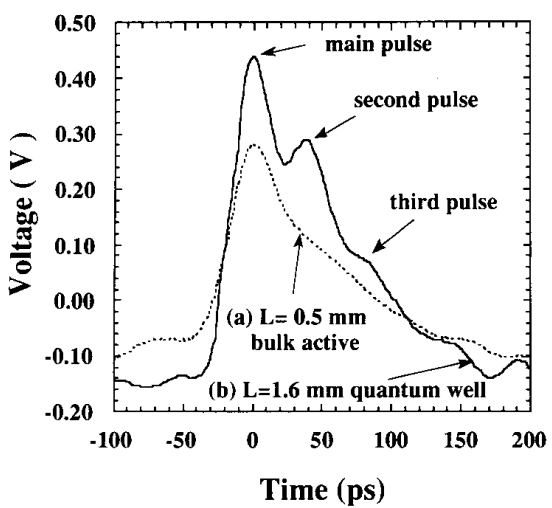

Fig. 5. (a) Absorber photocurrent versus time for the $500 \mu \mathrm{m}$ long bulk active region device with a $16 \mu \mathrm{m}$ saturable absorber length. (b) Absorber photocurrent versus time for a $1.6 \mathrm{~mm}$ long quantum well device with 80 $\mu \mathrm{m}$ saturable absorber.

\section{ACKNOWLEDGMENT}

The authors wish to acknowledge help from T. Reynold, W. B. Jiang, and P. Humphrey.

\section{REFERENCES}

[1] D. J. Derickson, P. A. Morton, R. L. Thornton, and J. E. Bowers, "A comparison of timing jitter in external and monolithic cavity mode-locked semiconductor lasers," Appl. Phys. Lett., vol. 59, Dec. 23, 1991.

[2] J. E. Bowers, P. A. Morton, A. Mar, and S. W. Corzine, "Actively mode-locked semiconductor lasers," IEEE J. Quantum Electron., vol. 56, p. 681,1989

[3] M. Schell, A. Weber, E. Schol, and D. Bimberg, "Fundamental limits of subpicosecond pulse generation by active mode-locking of semiconductor lasers: The spectral gain width and the facet reflectivities," IEEE J. Quantum Electron., vol. QE-27, p. 1661, 1991.

[4] C. Harder, J. S. Smith, K. Y. Lau, and A. Yariv, "Passive modelocking of a buried heterostructure laser with non-uniform current injection," Appl. Phys. Lett., vol. 42, p. 1020, 1983.

[5] G. P. Agrawal and N. A. Olsson, "Self-phase modulation and spectral broadening of optical pulses in semiconductor laser amplifiers," IEEE J. Quantum Electron., vol. QE-25, p. 2297, 1989.

[6] R. L. Thornton, W. J. Mosby, and T. J. Paoli, J. Lightwave Technology, vol. 6, p. 786, 1988. 